\title{
Design a methodology to model -reference control of first order delays system
}

\begin{abstract}
Design a nonlinear controller for second order nonlinear uncertain dynamical systems is one of the most important challenging works. This research focuses on the design, and analysis of a model-reference sliding mode controller for first order delay system, in presence of uncertainties. In order to provide high performance nonlinear methodology, model-reference sliding mode controller is selected. Pure sliding mode controller can be used to control of partly known nonlinear dynamic parameters. Conversely, pure sliding mode controller is used in many applications; it has an important drawback namely; chattering phenomenon. To attenuation the chattering, new filter based high speed control technique is introduced. In this technique, two type derivative techniques are used to improve the rate of delay as well stability, robustness and chattering attenuation. This technique cased to improve the rate of delay compare with conventional PID controller and conventional sliding mode controller.
\end{abstract}

Keyword: First order delays system; Model-reference sliding mode controller; Chattering phenomenon; Chattering attenuation; Filter-based sliding mode controller 